\section{Opinions regarding the health and welfare of dogs owned by homeless people}

\section{David Williams, Sarah Hogg}

Department of Veteriinary Medicine, University of Cambridge, Cambridge, Cambs, UK

Many consider that homeless people should not own dogs, since their health and welfare would not be adequate. Here we presented an online anonymous questionnaire to a group of veterinary surgeons, veterinary students and the general public asking questions regarding opinions on homeless people owning dogs.

Respondents were asked to identify their role, if any, in the veterinary profession, their gender and whether they were a pet owner. They were asked to grade their expectations of the physical health, body condition score (bcs) and behavioural issues in dogs belonging to homeless people (dhl) compared with dogs belonging to those living in homes (dh). Participants were asked if they gave money to the homeless, if this was done 'on the street' or via charitable organizations, and if they showed any bias towards or against homeless dog owners. Respondents were asked if they believed homeless people should be able to own dogs.

454 people replied to the questionnaire. 61\% expected the physical health of $\mathrm{dhl}$ to be lower than $\mathrm{dh}$. Three respondents expected the health of $\mathrm{dhl}$ to be better than that of $\mathrm{dh}$. $65 \%$ of respondents expected dhl to have a slightly lower bcs score than that of dh while $23 \%$ expected them to have a far lower bcs. 2 respondents expected the bcs of dhl to be higher than that of dh. $28 \%$ of respondents expected dhl to have slightly fewer behavioural issues than $\mathrm{dh}, 31 \%$ expected no difference and $28 \%$ expected dhl to have more behavior issues than dh. $43 \%$ of respòndents did not give money to homeless people, $42 \%$ gave money through charitable organisations, $15 \%$ gave to people on the street $8 \%$ without a preference for dog owners while $7 \%$ gave preferentially to dog owners. One respondent actively avoided giving money to homeless people owning dogs. $51 \%$ considered that homeless people lacked resources to adequately care for a pet dog. 58\% believed that dogs diverted money that could be better used. $93 \%$ agreed that dogs give homeless people companionship and $84 \%$ that dogs would improve their mental health.

The results of this study mirror attitudes held by many of the general public but differ from the results found in a study of the health and welfare of dhl. We hope that publishing both studies will improve attitudes towards dog ownership by homeless people.

\title{
Fireworks: don't leave it too late
}

\section{Jenny Newman ${ }^{1,4}$, Liam O'Brien², Aisling Mooney ${ }^{2}$, P-J.M. Noble ${ }^{1,3}$, Goran Nenadic ${ }^{4}$, Philip H. Jones', Rachel Casey ${ }^{5}$, Sophie Adamantos ${ }^{5}$, Alan D. Radford 6}

\author{
Epidemiology \& Population Health, University of Liverpool, Leahurst \\ Campus, Neston, UK \\ Nuffield Foundation Placement, Leahurst Campus, Neston, UK \\ School of Veterinary Science, University of Liverpool, Leahurst \\ Campus, Neston, UK \\ School of Computer Science, University of Manchester, Manchester, UK \\ University of Bristol, Bristol, UK \\ Department of Infection Biology, University of Liverpool, Leahurst \\ Campus, Neston, UK
}

The adverse impact of fireworks on animal behaviour is well recognised. Here we explore temporal trends and management of animals with fearful responses to fireworks presenting to veterinary surgeons.

The Small Animal Veterinary Surveillance Network (SAVSNET) collects data from consultations at participating veterinary practices in real-time. Electronic health records for 116,657 sick-animal consultations were collected between September 2012 and July 2014, including two traditional UK firework seasons celebrated annually on November 5th.

An open source, Python library for natural language processing was used to search the clinical notes from each consultation. This allowed us to identify all consultations that included consecutive pairs of words (bigram) or part words containing "fire" and "work" or "bon" and "fire", or common misspellings. In this manner, any consultation where fireworks or bonfire had been mentioned was retrieved. The free-text was then read to confirm the reason for presentation.

With this approach, 75 consultations were identified where assistance had been sought for the management of fear of fireworks. A further 17 consultations documented that an animal was fearful of fireworks, without a record of treatment having been sought.

Eighty-nine percent of those seeking advice for their pet's fear of fireworks in the three months prior to November 5 th (25/10,000 consultations), did so within one month of November 5 th $(49 \%$ within seven days, and $13 \%$ on November 5 th itself). For one third of those presenting within a week of November 5th, it was clear that the animals' responses to fireworks were known in advance.

The proportions of dogs, cats and other species in all consultations were $67 \%, 27 \%$ and $6 \%$, respectively, whilst in those consultations where treatment in anticipation of fearful responses was requested, the proportions were $97 \%, 3 \%$ and $0 \%$, respectively. In the 17 animals where help was not sought - but the animals were subsequently documented as being afraid of fireworks - the proportions were similar to that in the overall dataset.

In those animals receiving treatment, $52 \%$ received benzodiazepines, $25 \%$ pheromones and $41 \%$ nutraceuticals. In addition, $49 \%$ of consultations recorded providing animal behaviour advice.

The proximity of the vast majority of these consultations to November 5th, and the small number of consultations identified, highlights the need to raise awareness of the management strategies available to treat animals with noise-related fear, and which typically require several months of therapy. 